\title{
Herlyn-Werner-Wunderlich Syndrome Presenting with Abdominal Pain: A Case Report
}

\author{
Hayat Al-Jumah ${ }^{1 *}$, Rashida Sadiq1, Noor Al-Muslem², Fathia E. Al-Jama ${ }^{3}$, Raghad Aljishi', \\ Salah Abohelaika ${ }^{5}$
}

\author{
${ }^{1}$ Department of Obstetrics and Gynecology, Qatif Central Hosptial, Qatif, KSA \\ ${ }^{2}$ Department of Radiology, Qatif Central Hosptial, Qatif, KSA \\ ${ }^{3}$ King Fahad University Hospital, Private Health Services Centre, Khobar, KSA \\ ${ }^{4}$ Royal College of Surgeons, Dublin, Ireland \\ ${ }^{5}$ Clinical Pharmacology Department, Qatif Central Hosptial, Qatif, KSA \\ Email: `hayat.aljumah@gmail.com, rehman_sidhu@hotmail.com,n.f.muslem@gmail.com, \\ f_aljama@hotmail.com,raghad.aljishi@gmail.com,sabohelaika@moh.gov.sa
}

How to cite this paper: Al-Jumah, H., Sadiq, R., Al-Muslem, N., Al-Jama, F.E., Aljishi, R. and Abohelaika, S. (2021) Herlyn-Werner-Wunderlich Syndrome Presenting with Abdominal Pain: A Case Report. Open Journal of Obstetrics and Gynecology, 11, 911-916.

https://doi.org/10.4236/ojog.2021.117085

Received: June 20, 2021

Accepted: July 20, 2021

Published: July 23, 2021

Copyright $\odot 2021$ by author(s) and Scientific Research Publishing Inc. This work is licensed under the Creative Commons Attribution International License (CC BY 4.0).

http://creativecommons.org/licenses/by/4.0/ (c) (i) Open Access

\begin{abstract}
Herlyn-Werner-Wunderlich (HWW) syndrome is a rare congenital anomaly characterized by triad of uterus didelphys, obstructed hemi vagina, and ipsilateral renal agenesis. The most common presentation is abdominal pain, dysmenorrhea, and abdominal mass secondary to hemi-hematometrocolpos. We report an emergency presentation of this syndrome during the pandemic of COVID-19 in Qatif Central Hospital, Saudi Arabia, April 2020. An 11-year-old Saudi girl presented to the emergency room with three months history of cyclical lower abdominal pain. The pain was progressive, continuous and not relieved by analgesics. Abdominal examination revealed a tender abdominal mass mainly in the left iliac fossa, Ultrasound evaluation showed two uterine bodies. The left uterus was distended with complex fluid. Pelvic MRI findings consistent with Herlyn-Werner-Wunderlich (HWW) syndrome were found with uterine didelphys and left sided hematometra resulting from obstructed hemi-vagina and ipsilateral agenesis of the left kidney. Resection of the vaginal septum and drainage of hematometra was done. The patient recovered with normal cyclical menstruation. Cyclical or continuous lower abdominal pain with or without amenorrhea is the usual presentation of HWW syndrome during adolescence. Diagnosis is made by ultrasonography and MRI. Early diagnosis and accurate management can provide pain relief, prevent future complications, and preserve fertility.
\end{abstract}

\section{Keywords}

Herlyn-Werner-Wunderlich Syndrome, Obstructed Hemi-Vagina, 
Hematometrocolpos, Mullerian Anomaly, Vaginoplasty

\section{Introduction}

Herlyn-Werner-Wunderlich (HWW) syndrome is characterized by triad of uterus didelphys with obstructed hemi-vagina and ipsilateral renal agenesis, and it is a rare variant of para mesonephric (Mullerian) duct anomaly [1]. Obstructed hemi-vagina and ipsilateral renal agenesis (OHVIRA) syndrome is another name [2]. It is usually presented with abdominal pain, dysmenorrhea and abdominal mass secondary to hematocolpos [3]. In 1922 Purslow first described this syndrome in a young woman who presented with gradually increasing pelvic pain and a pelvic mass with regular menstruation [4].

We investigated the case of a young girl with triad of obstructed hemi-vaginal, uterus didelphys, and left renal agenesis. She was suffering from abdominal pain at the age of menarche and successfully managed by transvaginal resection of the vaginal septum after diagnosis by pelvic ultrasound and MRI.

\section{Case Report}

A Saudi girl aged 11 years was brought to the emergency unit with three months history of episodic lower abdominal and rectal pain. The pain was progressive, continuous and was not relieved by analgesics. Her age of menarche was 10 years with regular menses every 28 days for 3-5 days, but with scanty flow associated with severe dysmenorrhea and difficulty defecating. Her birth was at term and uneventful. She had no past medical or surgical history of any diseases. Her development was normal with no family history of congenital disease. Her parents were non-consanguineous.

The girl was in pain upon arriving at the hospital. Her vital signs and general physical examination were unremarkable. The abdomen was distended with a palpable pelvi-abdominal mass of $4 \mathrm{~cm}$ above the symphysis pubis, tender and shifted to the left iliac fossa. There were no signs of peritonitis nor hepatosplenomegaly. The perineal inspection confirmed an intact and normal hymen.

Blood and urine tests and pelvic ultrasound were done. Her hemoglobin was $12.4 \mathrm{mg} / \mathrm{dl}$ and blood and urine tests came back normal. Ultrasound revealed two endometrial cavities, one of them is distended and filled with heterogeneous internal echoes. The left kidney was not seen. Appearance raised the suspicion of didelphys uterus with hematometra, hematocolpos, and agenesis of the left kidney. Further, evaluation by pelvic MRI was done and showed two widely separated uterine horns and two cervices, consistent with uterus didelphys with an apparent one vagina Figure 1(a). The left uterine horn and the left cervix were distended and filled with blood and clots, consistent with hematometra and hematocervix Figure 1(b). There was an intramural hematoma in the left cervical wall communicating with the endocervix and measuring $4.8 \times 2.4 \mathrm{~cm}$ 
(Figure 2). The right uterine horn was normal in size with preserved zonal anatomy and normal endometrium, which appeared compressed in the lower part by the left hematocervix. Both ovaries were normal. The left kidney was not visualized in the left renal fossa or in an ectopic location with a normal solitary right kidney compatible with left renal agenesis Figure 3. The other imaged abdominal organs were grossly normal.

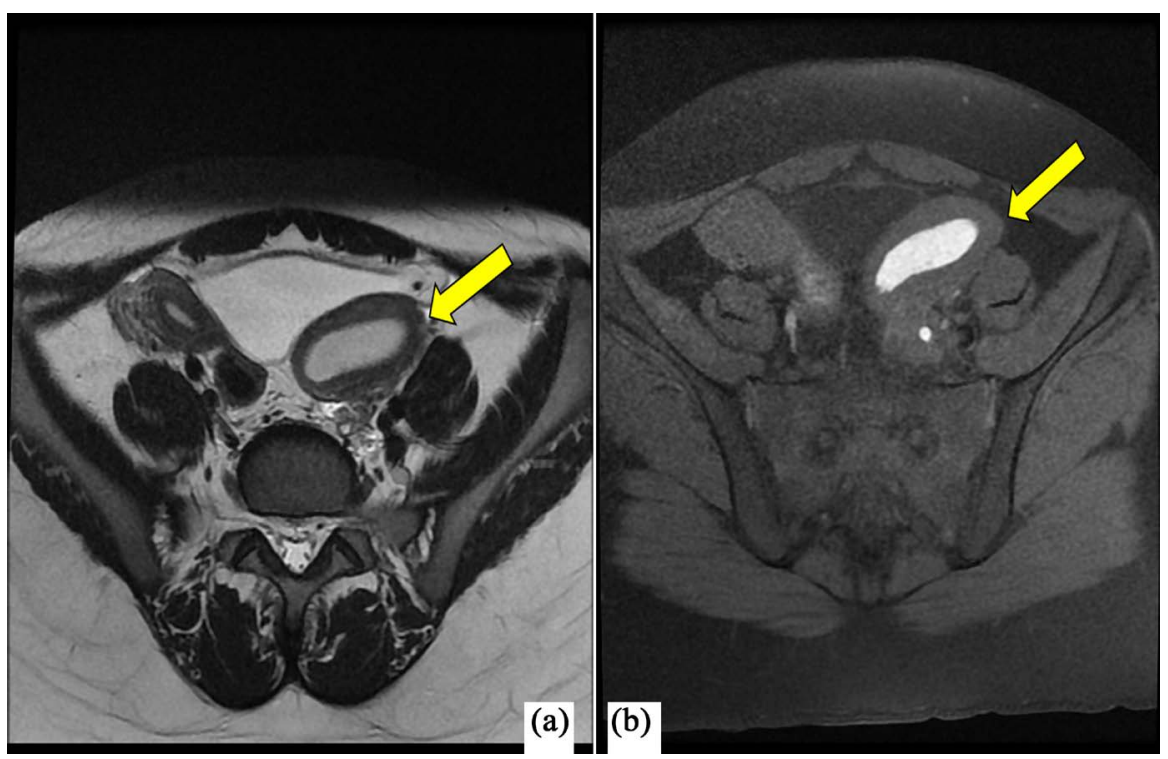

Figure 1. MRI of the pelvis (a) Axial T2WI demonstrating uterus didelphys with distended left uterine horn and normal right one. (b) Axial T1 FATSAT showing bright intrauterine signal intensity consisting with hematometra.

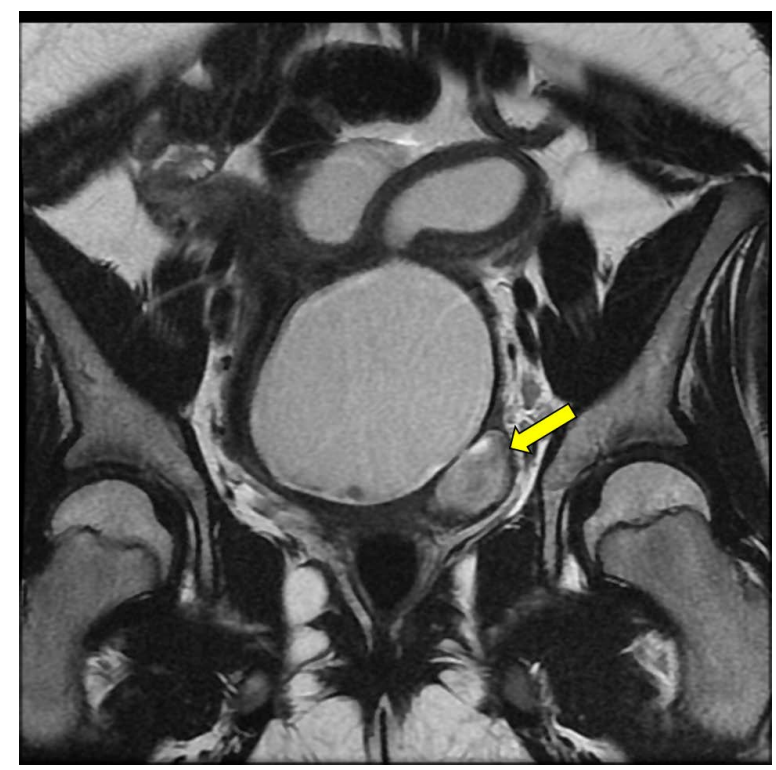

Figure 2. MRI of the pelvis, coronal T2WI, demonstrating distended left uterine horn and left cervix filled with high T2 and T1 (not shown) signal intensity with dark signal in the dependent part suggestive of hematometra and hematocervix with clots. There is small intramural hematoma in the left lateral cervix communicating with the left hematocervix (yellow arrow). 


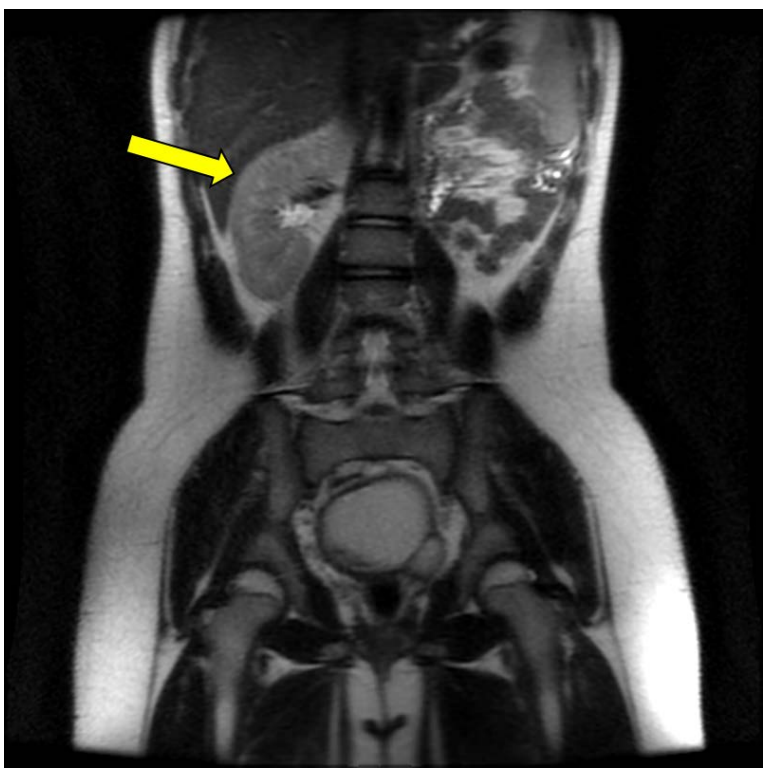

Figure 3. Coronal SSFSE MRI of the abdomen and pelvis demonstrating absence of the left kidney and normal right one.

\section{Surgical Approach}

A huge amount of tarry blood was drained by reaching up to the left cervix after identification and resection of the left blind fold of the vaginal septum. The vaginal canal was reconstructed. A French-20 gauge Foley's catheter was left in the opened left blind vaginal fold and filled with $50 \mathrm{cc}$ saline to keep it in place for free drainage of blood and to keep the vaginal fold open. In addition, packing was done with roller gauze in the resected vaginal fold beside the balloon of catheter and one end of roller gauze was left out at the introitus. There were no pre- or post-operative complications. Vaginal packing was removed 12 hours post-surgery. The patient was discharged on the third post-operative day with a drain in place to be kept for two weeks, and she was given oral antibiotics. The drain was removed two weeks later and the patient subsequently developed monthly regular menstruation after that.

\section{Discussion}

HWW syndrome (obstructed hemi-vagina with ipsilateral renal agenesis) was firstly reported in 1971 by Herlyn and Werner [5]. The association of the right renal agenesis with a bicornuate uterus and single vagina in the presence of isolated hematocervix was described by Wunderlich in 1976 [5].

Mullerian duct abnormalities (MDA) are estimated to be prevalent in 2\% - 3\% of women. Some $43 \%$ of these cases are associated with renal anomalies, and uterus didelphys constitute $11 \%$ [6]. The vaginal septum in HWW is commonly longitudinal; however, the majority of didelphys patients have either a complete or partial vaginal septum [3] [5] [7].

Internal urinary tract and genital organs come from two paired mesonephric (Wolffian) and paramesonephric (Mullerian) ducts. Wolffian ducts are also 
component elements for adequate fusion of Mullerian ducts. If one of the Wolffian ducts is absent, the ipsilateral kidney and ureter will fail to fuse in the midline and might be complete or incomplete [5]. However, HWW syndrome's precise etiopathogenesis is still unknown [5] [8]. From the paired paramesonephric ducts, the uterus, fallopian tube, cervix, and upper two-thirds of the vagina develop. It runs lateral to the mesonephric duct posteriorly and downwards and comes closer to the paramesonephric duct in the midline from the opposite side and their fusion results in the formation of the uterus, cervix and upper part of the vagina. A didelphys results when they fail to fuse in the midline [5]. Zhu et al have provided a new classification of HWW syndrome according to the completeness of vagina obstruction into complete and incomplete obstructed hemi-vagina [8].

Due to the rare occurrence of HWW syndrome, the diagnosis may be delayed in the event of an incomplete hemi-vaginal obstruction and a normal menstrual flow from the non-obstructed side [2] [9]. Dysmenorrhea is the main symptom of HWW syndrome and is usually established after puberty [2] [10]. The increase in pain is related to a rise in the volume of hematocolpos caused by an obstructed hemivagina [2] [10] [11]. Endometriosis, inflammation, twisted cysts, and appendicitis are other differential causes of pelvic pain and must be excluded [2] [10]. HWW syndrome's consequences and fertility dysfunction could be reduced by early diagnosis of endometriosis in such patients [1] [10] [11]. Other uncommon presentations are urinary retention and difficulty in defecation due to pressure of hematocolpos on the urethra or rectum [10].

Ultrasonography and MRI are useful in diagnosing this condition [3] [5] [10]. MRI is considered an outstanding criterion for diagnozing and preoperative planning of HWW syndrome [5] [10]. Uterine morphology, detection of communication between vaginal and uterine lumen, nature of fluid contents, renal agenesis, and complications like endometriosis can also be diagnosed by MRI [5]. MRI has the ability for multi planner imaging without the radiation hazard. The recommended treatment for obstructed hemi-vagina with hematocolpos is the resection of the vaginal septum [3] [10] [11]. The hysteroscopic approach is usually used for vaginal septotomy. Since the cervical agenesis is difficult to be corrected surgically, laparoscopic or trans-abdominal resection of the involved ipsilateral uterus and fallopian tube is proposed [3] [5] [10].

\section{Conclusion}

The onset and presentation of HWW syndrome vary. Ultrasonography is considered a low-cost, real-time, and economic tool for diagnosing HWW syndrome. On the other hand, MRI offers detailed and multi planner imaging with no radiation hazards. Early surgical intervention is crucial for overcoming the internal genital tract obstruction and for reducing the risk of infertility and endometriosis. Early diagnosis and treatment of HWW syndrome have a good prognosis. Increasing awareness of this syndrome amongst general practitioners and healthcare professionals is necessary for preventing misdiagnosis of this condition. 


\section{Funding Source}

There was no funding source for this paper.

\section{Acknowledgements}

We thank the patient and her family for their consent to publish this article.

\section{Conflicts of Interest}

The authors declare no conflicts of interest regarding the publication of this paper.

\section{References}

[1] Orazi, C., Lucchetti, M.C., Schingo, P.M., Marchetti, P. and Ferro, F. (2007) Herlyn-Werner-Wunderlich Syndrome: Uterus Didelphys, Blind Hemivagina and Ipsilateral Renal Agenesis. Sonographic and Mr Findings in 11 Cases. Pediatric Radiology, 37, 657-665. https://doi.org/10.1007/s00247-007-0497-y

[2] Jia, G., Chai, W., Cui, M., Wen, Y., Cui, L. and Gong, F. (2018) A Case Report on Herlyn-Werner-Wunderlich Syndrome with Spontaneous Abortion. Medicine, 97, e12004. https://doi.org/10.1097/MD.0000000000012004

[3] Wang, J., Zhu, L., Lang, J., Liu, Z., Sun, D., Leng, J., et al. (2014) Clinical Characteristics and Treatment of Herlyn-Werner-Wunderlich Syndrome. Archives of Gynecology and Obstetrics, 290, 947-950. https://doi.org/10.1007/s00404-014-3286-5

[4] Purslow, C. (1922) A Case of Unilateral Hæmatokolpos, Hæmatometra and Hæmatosalpinx. BJOG: An International Journal of Obstetrics and Gynaecology, 29, 643-643. https://doi.org/10.1111/j.1471-0528.1922.tb16100.x

[5] Khaladkar, S.M., Kamal, V., Kamal, A. and Kondapavuluri, S.K. (2016) The Herlyn-Werner-Wunderlich Syndrome: A Case Report with Radiological Review. Polish Journal of Radiology, 81, 395-400. https://doi.org/10.12659/PJR.897228

[6] Lee, B.H., Kim, J.W., Oh, S.I., Kim, M.H., Park, N.H., Lee, J.Y., et al. (2001) 3 Cases of Uterus Didelphys with an Obstructed Hemivagina and Ipsilateral Renal Agenesis. Korean Journal of Obstetrics \& Gynecology, 40, 1489-1495.

[7] Heinonen, P.K. (2000) Clinical Implications of the Didelphic Uterus: Long-Term Follow-Up of 49 Cases. European Journal of Obstetrics Gynecology and Reproductive Biology, 91, 183-190. https://doi.org/10.1016/S0301-2115(99)00259-6

[8] Zhu, L., Chen, N., Tong, J.-L., Wang, W., Zhang, L. and Lang, J.-H. (2015) New Classification of Herlyn-Werner-Wunderlich Syndrome. Chinese Medical Journal, 128, 222. https://doi.org/10.4103/0366-6999.149208

[9] Epelman, M., Dinan, D., Gee, M.S., Servaes, S., Lee, E.Y. and Darge, K. (2013) Müllerian Duct and Related Anomalies in Children and Adolescents. Magnetic Resonance Imaging Clinics of North America, 21, 773-789. https://doi.org/10.1016/j.mric.2013.04.011

[10] Widyakusuma, L.S., Lisnawati, Y., Pudyastuti, S. and Haloho, A.H. (2018) A Rare Case of Pelvic Pain Caused by Herlyn-Werner-Wunderlich Syndrome in an Adult: A Case Report. International Journal of Surgery Case Reports, 49, 106-109. https://doi.org/10.1016/j.ijscr.2018.06.022

[11] Santos, X.M. and Dietrich, J.E. (2016) Obstructed Hemivagina with Ipsilateral Renal Anomaly. Journal of Pediatric and Adolescent Gynecology, 29, 7-10. https://doi.org/10.1016/j.jpag.2014.09.008 